\title{
BIOMARKERS OF DISEASE ACTIVITY IN SYSTEMIC SCLEROSIS
}

10.36740/WLek202010137

\author{
Katarzyna Karina Pawlik', Anna Bohdziewicz', Magdalena Chrabąszcz² , Anna Stochmal2 , Mariusz Sikora², \\ Rosanna Alda-Malicka', Joanna Czuwara², Lidia Rudnicka² \\ 'STUDENT RESEARCH COMMITTEE, DEPARTMENT OF DERMATOLOGY, MEDICAL UNIVERSITY OF WARSAW, WARSAW, POLAND \\ 2DEPARTMENT OF DERMATOLOGY, MEDICAL UNIVERSITY OF WARSAW, WARSAW, POLAND
}

\begin{abstract}
Systemic sclerosis (SSc) is an autoimmune disease characterized by vasculopathy and uncontrolled cutaneous and internal organs fibrosis. Diagnosis of SSc in an early phase can be difficult because of a lack of typical symptoms. The delay in diagnosis and treatment of SSc may lead to uncontrolled progression of the disease, thus identification of possible early indicators of skin and organ involvement to prevent their further damage is necessary. The aim of this study is to review the latest biomarkers of organ involvement in SSc. In patients with lung fibrosis lung-epithelial-derived surfactant protein (SP-D), the glycoprotein Krebs von den Lungen-6 (KL-6), and chemokine ligands 2, 4 and 18 (CCL2, CXCL4, CCL18) are elevated, while in patients with skin fibrosis serum levels of heat shock protein 27 (Hsp27), interleukin 16 (IL-16), and lgG-galactosylation ratio are increased. Adiponectin concentration is inversely correlated with the intensity of cutaneous fibrosis. Skin gene profiling also seems very promising. In patients with heart involvement increased serum levels of brain natriuretic peptide (BNP) are present, as well as raised Midkine and Follistatin-like 3 (FSTL3) proteins, ratios of Cu/Se and ceruloplasmin(CP)/Circulating selenoprotein P(SELENOP) and higher whole blood viscosity level. Elevated calprotectin levels are found in individuals with gastrointestinal involvement. Increased levels of chemerin and ARA autoantibodies are associated with renal involvement, whereas high levels of adhesion molecules are found in patients with scleroderma renal crisis (SRC). Currently there are no biomarkers in use that can specifically identify the early involvement of organs.
\end{abstract}

KEY WORDS: systemic sclerosis, fibrosis, autoantibodies, biomarker

Wiad Lek. 2020;73(10):2300-2305

\section{INTRODUCTION}

Systemic sclerosis (SSc) is an incurable autoimmune disease characterized by vasculopathy, specific autoantibodies, and both cutaneous and organ fibrosis. Excessive deposition of collagen can result in irreparable scarring of the vital organs leading to their failure [1] and affecting the length and quality of life [2]. The pathogenesis of SSc is multi-faceted and remains a complex research object. SSc can be split into two subsets based on the intensity of skin hardening: limited cutaneous SSc (lcSSc) and diffuse cutaneous SSc ( $\mathrm{dcSSc})$. LcSSc is less severe form and is rarely connected with internal organ manifestations except for late-onset pulmonary arterial hypertension; skin lesions are located distally to the elbow and knee joints. DcSSc is characterized by skin thickening which is both proximal and distal to the elbows and knees. It is a more serious type associated with organ involvement such as scleroderma renal crisis, interstitial lung disease, and myocardial fibrosis $[1,3]$.

Lung changes in SScinclude both extrapulmonary features as well as intrapulmonary manifestations. Lung fibrosis and pulmonary hypertension $(\mathrm{PH})$ are the most life-threatening complications in SSc and have notable effects on disease outcomes. $\mathrm{PH}$ is a condition of increased blood pressure within the arteries of the lungs. It is caused by pulmonary vasculopathy or develops secondary to pulmonary fibrosis (also known as interstitial lung disease (ILD)) [4].
Gastrointestinal symptoms may accompany both diffuse and limited forms of SSc. Abnormalities in the functioning of the gastrointestinal tract are present in almost $90 \%$ of patients [5], and can occur even when skin fibrosis is absent [6]. As far as the internal organs are concerned, the most frequently involved organ is the oesophagus. Microstomia, xerostomia, gastroesophageal reflux disease (GERD), lower oesophageal sphincter (LOS) abnormalities, gastroparesis, gastric antral vascular ectasia (GAVE), chronic intestinal pseudo-obstruction (CIPO), small intestinal bacterial overgrowth (SIBO), and fecal incontinence seem to be the most important digestive system diseases connected with the presence of SSc [5].

Renal involvement can occur as an asymptomatic reduction of glomerular filtration rate (GFR), increased renal resistance indices, vasculitis associated with ANCA, or as life-threatening scleroderma renal crisis (SRC), which according to the latest data has become quite a rare complication in SSc patients. Nevertheless, SRC is a significant cause of morbidity and mortality in which risk factors are still poorly understood $[1,7]$.

Cardiac manifestations of SSc can be divided into direct (progressive heart muscle inflammation and fibrosis) and indirect - connected with the impaired function of other organs (pulmonary arterial hypertension, renal crisis) [8]. It is estimated that during the disease about $12 \%$ of patients with SSc will develop pulmonary arterial hypertension (PAH) [9]. 
Diagnosis of SSc in an early phase is difficult because of the lack of typical signs and symptoms. The early detection and treatment of patients who have a high risk of disease progression is crucial to avoid organs disability in future. Unfortunately currently there are no biomarkers in use that can identify the early involvement of organs.

\section{THE AIM}

The aim of the study is to review the latest biomarkers of organ involvement in SSc reported in the literature.

\section{REVIEW}

\section{BIOMARKERS IN SYSTEMIC SCLEROSIS}

\section{SKIN}

Nowadays, the gold standard in the assessment of skin involvement is the modified Rodnan skin score (mRSS). It consists of palpation and pinching of the skin within 17 anatomical areas and assessment of skin thickness on a four-stage scale (0 to 3 ) within each of them. It is a non-invasive and inexpensive method, however it is considered to be subjective and depends on experience of the examining doctor. There is a need to find biomarkers that correlate with mRSS and can be objectively measurable.

Studies regarding heat shock protein 27 (Hsp27), which serves as a pro-inflammatory signaling molecule and helps the cell to survive under conditions of stress have been conducted. In Japanese study, serum levels of Hsp27 in 67 SSc patients were investigated, it was found that the serum levels of Hsp27 correlated positively with mRSS and were notably higher in patients with dcSsc than in those with lcSSc or healthy controls [10].

Another potential biomarker which has recently been investigated is the IgG-galactosylation ratio (IgG-Gal ratio), which has been found to be increased in immune diseases and certain cancers. In Chinese patients with SSc and healthy controls the IgG-Gal ratio was measured. Results showed that the IgG-Gal ratio was positively associated with mRSS and thus may be useful in assessing the severity of skin fibrosis and helpful in distinguishing between SSc subtypes [11].

Another marker worthy of attention is adiponectin, a hormone produced and secreted into the blood by activation of the nuclear receptor PPAR- $\gamma$ which affects many metabolic processes. It was discovered that the level of adiponectin is inversely proportional to skin fibrosis and correlates negatively with the modified Rodnan skin score $[12,13]$.

Expression of IL-16 in SSc lesions and patients sera has also been examined. The correlation between serum IL-16 levels and the clinical symptoms of SSc in the skin was investigated. According to the results, the percentage of IL-16-positive cells was higher in patients with dcSSc than in healthy individuals. Patients with dcSSc also had increased serum levels of IL-16. Consequently, it has been suggested that IL-16 serum concentration may serve as biomarker to determine the severity of skin fibrosis [14].
In order to discover novel biomarkers scientists have started to study patients genes. Thus far the expression of genes regulated by TGF $\beta$ and IFN $\gamma$ has been taken into account. Analysis of skin samples from patients with dcSSc revealed a four-gene biomarker panel consisting of THBS1, COMP, SIGLEC1, and IFI44; their expression correlated moderately well with mRSS $[15,16]$. Another study discovered a two-gene pharmacodynamic biomarker (THBS1, MS4A4A) which may prove useful in the longitudinal assessment of skin involvement [17]. Recent studies have delved into the topic of epigenetics, including long noncoding RNAs (lncRNAs), increased plasma levels of TINCR, HOTTIP, and SPRY4-IT1 and decreased levels of ANCR were observed in SSc patients in comparison with the control group. Additionally, HOTTIP and SPRY4-IT1 were positively correlated with mRSS. ANCR and SPRY4-IT1 also correlated with PAH. Plasma SPRY4-IT1, HOTTIP, ANCR, and TINCR appear to be candidates for SSc biomarkers and SPRY4-IT1 may be used to predict the risk of SSc and define subtype [18]. These studies suggest that there is scope for more effective risk stratification and treatment selection for patients with SSc based on the expression of skin genes profiles.

\section{LUNGS}

Several molecules have been considered as biomarkers of lung involvement and many studies indicate that they may be very useful in the future.

It is important to mention endothelial microparticles (EMP) - submicron vesicles released in healthy and disease conditions from different cells which may modulate cell-cell signaling in vascular diseases. It was discovered that CD144+ EMP levels were significantly higher in the SSc-PAH patients. Moreover, the group of EMP molecules in SSc patients was more distinct [19]. EMP (especially CD144+) are potentially promising biomarkers of SSc-PAH and may be involved in its pathogenesis.

According to one study, in which the levels of lung-epithelial-derived surfactant protein (SP-D) and the glycoprotein Krebs von den Lungen-6 (KL-6) were assessed in SSc patients serum samples, SP-D is a relevant diagnostic biomarker of SSc-associated interstitial lung disease, whereas KL-6 could assess the severity of lung fibrosis [20].

The large groups of molecules which may be considered as biomarkers of lung changes are chemokine ligands and interleukins. Chemokine (C-C motif) ligand 2 (CCL2), also called monocyte chemoattractant protein-1 (MCP-1), is released by a variety of immune cells and participates in the migration and activation of monocytes and $\mathrm{T}$ cells. It was reported that elevated serum CCL2 levels are associated with the presence of ILD and correlated with its severity [21]. Chemokine (C-X-C motif) ligand (CXCL4), known as platelet factor 4 (PF-4), plays a key role in cell migration, inflammation and wound repair; plasma CXCL4 levels were elevated in SSc patients and were associated with lung fibrosis and $\mathrm{PAH}$ [22]. Chemokine (C-C motif) ligand 18 (CCL18) is selectively chemotactic for $\mathrm{T}$ cells and has been shown to directly stimulate collagen production in fibroblasts [20]. It has been reported that serum CCL18 levels were elevated in SSc patients - according to a number of studies CCL18 appeared as a strong prognostic 
marker for SSc-associated ILD progression as well as predictive biomarker for worsening of the lung disease in $\operatorname{SSc}[3,20]$.

Results of some analyses have indicated that serum OX40L levels were elevated in SSc patients, especially in those diagnosed with dcSSc [23], but the latest studies suggest there is no significant association [20].

It has been suggested that the most solid biomarkers for diagnosis, classification, predicting organ involvement and prognosis in SSc are antinuclear autoantibodies. Each type of antibody is connected with a different SSc subset and is strongly predictive of the specific organ manifestations [24]. Anti-topoisomerase I antibodies are associated with ILD - it was reported that the presence of these antibodies correlated with a decline in differential forced vital capacity (FVC) levels [25]. Focusing on advances in the SSc antibody-biomarker field, recent studies have shown monospecific anti-Ro52/ tripartite motif-containing 21 (TRIM21) antibodies are associate with ILD and poor survival in SSc [26]. Moreover, many functional autoantibodies against cell surface receptors have also been identified in SSc. Specifically autoantibodies against the angiotensin II type 1 receptor (AT1R) and the endothelin-1 type A receptor (ETAR) have been reported as being predictive and prognostic biomarkers of SSc-PAH [27].

\section{GASTROINTESTINAL TRACT}

In SSc, only the skin is affected more often than the gastrointestinal tract [28]. Recent studies reported the role of fecal calprotectin (FC) as a potential biomarker of small intestinal bacterial overgrowth (SIBO). FC levels are elevated during active inflammation of the intestines. Measurement is uncomplicated and non-invasive, which makes it a very useful prospective test. A cohort study of 125 patients showed that elevated FC levels $(>50 \mathrm{mcg} / \mathrm{g})$ were related to the severity of clinical digestive symptoms, delayed gastric emptying, and esophageal motor involvement. A strong association was reported between the presence of SIBO and a highly elevated FClevels $(>275 \mathrm{mcg} / \mathrm{g})$. This test may help to identify a group of high-risk SSc patients, who require a glucose breath test - the assessment traditionally used to diagnose SIBO. The measurement of FC levels can also be used to evaluate the eradication of SIBO [29].

In patients with interstitial lung disease, the concentration of FC has also been found to increase. The role and usefulness of this observation in the early diagnosis of ILD are currently being investigated [30].

Presently available treatment of GI involvement is symptomatic and has short-time efficacy. No disease-modifying drugs are yet known. Identification of novel biomarkers could be helpful both for earlier diagnosis and the research of effective medications [6].

\section{KIDNEYS}

The kidneys are commonly involved in dcSSc. It was discovered that there is a strong association between scleroderma renal crisis (SRC) and anti-RNA polymerase III autoantibodies. Genetic susceptibility and altered protein expression were analyzed in kidney biopsy specimens from anti-RNAP III-positive SRC patients. The expression of two proteins - GPATCH2L and CTNND2- were increased in SRC in comparison with control samples. This may indicate the potential role of these proteins in the pathogenesis of SRC [31]. Findings reported by Liu et al. also demonstrated that anti-RNA polymerase III antibodies have predictive value for SSc-related renal crisis [32].

Additionally, elevated serum levels of circulating molecules: intercellular adhesion molecule-1 (ICAM-1), vascular cell adhesion protein-1 (VCAM-1) and E-selectin have also been found in patients with SRC [1].

It is also important to mention that biomarkers of activation of complement pathways were significantly elevated in SSc in comparison to healthy patients, and were similar to those found in other rheumatic diseases. Deposits of C3b molecules in some patients with SRC were also found [33]. Studies have shown that elevated levels of chemerin were related to an increased risk of renal injury, this may be due to direct damage of the kidneys or decreased chemerin clearance both in patients with SSc as well as in general population [13].

\section{HEART}

Right heart catheterization (RHC) is required to diagnose $\mathrm{PAH}$. Due to invasiveness of this procedure, it is usually performed only in high-risk patients. Identification of possible indicators of heart involvement that would be appropriate for non-invasive screening is necessary. That will help to reduce the need for RHC and may lead to better therapeutic outcomes [34]. In clinical practice, the DETECT algorithm for $\mathrm{PAH}$ detection is commonly used, one of its components is the measurement of serum levels of the $\mathrm{N}$-terminal fragment of pro-brain natriuretic peptide (NT-proBNP). This marker is elevated in SSc patients with PAH, but it is not specific for PAH or right ventricular dysfunction. It can also be increased in left heart dysfunction or in renal insufficiency [3, 35]. NT-proBNP appears to be a credible and independent predictor of mortality in patients with SSc [36], the level of this marker is also associated with skin fibrosis and is higher in patients with the diffuse rather than limited type of SSc [8].

Moreover, a negative correlation between the level of miRNA let-7d and PAH has been observed, suggesting a potential role of miRNA let-7d as a biomarker for the presence and severity of PAH in SSc [37].

In addition, two proteins, Midkine and Follistatin-like 3 (FSTL3) combined, may act as SSc-PAH indicators and have been identified as potential drug targets [34].

Recent studies have shown that patients with SSc-related $\mathrm{PAH}$ have elevated ratios of $\mathrm{Cu} / \mathrm{Se}$ and ceruloplasmin $(\mathrm{CP})$ /Circulating selenoprotein $\mathrm{P}(\mathrm{SELENOP})$ when compared to controls - this implies that these parameters may help to identify the risk of SSc-related PAH. Furthermore, a shortage of selenium in patients with skin fibrosis may indicate a deficiency of selenzymes needed to slow down the pathogenesis of scleroderma and successful control of oxidative stress [38].

A recent cohort study showed that higher whole blood viscosity levels in SSc patients can serve as an independent indicator for PAH development. However, this needs to be confirmed by further research [39]. 
Table 1. Potential biomarkers of organ involvement in SSc patients.

ORGAN

SKIN

BIOMARKER

Heat shock protein 27

IgG-galactosylation ratio

Adiponectin

Interleukin 16

THBS1, COMP, SIGLEC1 and IFI44 four-gene signature

THBS1 and MS4A4A two-gene signature

SPRY4-IT1, HOTTIP, ANCR, and TINCR

GASTROINTESTINAL TRACT

Fecal calprotectin

NT-proBNP

miRNA let-7d

HEART

Midkine and Follistatin-like 3 (FSTL3)

Ratios of Cu/Se and CP (ceruloplasmin)/SELENOP(Circulating selenoprotein $\mathrm{P}$ )

Whole blood viscosity level

CD144+ EMP

SP-D and KL-6

CCL2, CCL18, CXCL4

LUNGS

IL-6

Endothelin-1

Autoantibodies: Anti- Ro52/TRIM, Agonistic anti-AT1R, Agonistic anti-ETAR, Anti-topoisomerase I, Anti-Th/To

\begin{tabular}{|c|c|}
\hline \multirow{5}{*}{ KIDNEYS } & anti-RNAP III autoantibodies \\
\hline & ICAM-1, VCAM-1 \\
\hline & GPATCH2L and CTNND2 \\
\hline & C3b molecules \\
\hline & Chemerin \\
\hline
\end{tabular}

NT-proBNP - N-terminal fragment of pro-brain natriuretic peptide; EMP- endothelial microparticles; SP-D - lung-epithelial-derived surfactant protein; KL-6- glycoprotein Krebs von den Lungen-6; CCL2-Chemokine (C-Cmotif) ligand 2 = monocyte chemoattractant protein-1 (MCP-1); CXCL4-Chemokine (C-X-C motif) ligand = platelet factor 4 (PF-4); CCL18 - Chemokine (C-C motif) ligand 18; Anti-R052/TRIM - anti-Ro52/tripartite motif-containing 21 antibodies; Agonistic anti-AT1R-autoantibodies against the angiotensin II type 1 receptor; Agonistic anti-ETAR - autoantibodies against the endothelin-1 type A receptor; anti-RNAP III autoantibodies - anti-RNA polymerase III autoantibodies; ICAM-1 - intercellular adhesion molecule-1; VCAM-1-vascular cell adhesion protein

\section{DISCUSSION}

The main aim of this review is to discuss the latest biomarkers of organ changes in SSc and underline their importance.

Taking into account the results of the search for biomarkers correlating with mRSS the most recent are heat shock protein 27, IgG-galactosylation ratio, and IL-16 $[10,11,14]$. It also appears to be very promising to study skin gene profiles of SSc patients, including epigenetics (Table 1) [15-18].

Results of one of the latest studies appear to show that the serum level of KL-6 may reliably assess ILD severity to optimize further patient's management, while the serum level of SP-D is useful for early identification of SSc patients having developed ILD. The same study also showed that there is no confirmation that the serum level of OX40L may be associated with lung fibrosis [20]. Many studies have proven the levels of chemokines (CCL2, CCL18, CXCL4) are reliable biomarkers for activity as well as severity of the lung fibrosis (Table 2) [20-22].
Measurement of fecal calprotectin level seems to be useful in identifying SSc patients at high risk of gastrointestinal tract involvement [29].

In case of renal involvement, even though SRC has become quite a rare complication in SSc patients, most of the research is focused on this life-threatening complication $[7,40]$. The latest research supports a potential role for altered Wnt signaling in SRC pathogenesis and indicates that anti-RNA polymerase III autoantibodies seem to be one of the most promising biomarker of renal involment in SSc [31,32] as well as chemerin, which high level is associated with renal function impairment [13].

In diagnosis of patients with $\mathrm{PAH}$ the measurement of NT-proBNP is already in use [35]. However, the search for more specific markers is in progress. The most recent studies have concerned expression of miRNA let-7d, Midkine and Follistatin-like 3 (FSTL3) proteins, ratios of $\mathrm{Cu} / \mathrm{Se}$ and ceruloplasmin (CP) /Circulating selenoprotein P(SELENOP), and whole blood viscosity level [34,37-39]. (Table 1) 
Table 2. SSc lung changes with potential biomarkers.

\begin{tabular}{ccc}
\hline Biomarkers & Association & Value of the biomarker \\
\hline Microparticles & & \\
\hline EMP CD144+ & PAH & Predictive \\
\hline Proteins & & \\
\hline SP -D & Lung fibrosis & Diagnostic \\
KL -6 & Lung fibrosis & Severity \\
\hline Chemokines & Lung fibrosis & \\
\hline CCL18 & Lung fibrosis & Predictive, Prognostic \\
CCL2 & Sung fibrosis, PAH & Peverity, Activity \\
CXCL4 & & Predictive \\
\hline Interleukins & Lung fibrosis & \\
\hline IL-6 & PAH & Predictive \\
\hline Endothelin-1 & & Severity \\
\hline Autoantibodies & ILD & Prognostic \\
\hline Anti-Ro52/TRIM21 & PAH & Predictive, Prognostic \\
Agonistic anti-AT1R & PAH & Predictive, Prognostic \\
Agonistic anti-ETAR & Predictive \\
Antitoposomerase I & Predictive \\
Anti-Th/To & ILD, Lung fibrosis & ILD, Lung fibrosis \\
\hline
\end{tabular}

EMP- endothelial microparticles; SP-D - lung-epithelial-derived surfactant protein; KL-6 - glycoprotein Krebs von den Lungen-6; CCL2 - Chemokine (C-C motif) ligand 2 = monocyte chemoattractant protein-1 (MCP-1); CXCL4 - Chemokine (C-X-C motif) ligand = platelet factor 4 (PF-4); CCL18 - Chemokine (C-C motif) ligand 18; IL-6 - Interleukin 6; Anti- Ro52/TRIM - anti-Ro52/tripartite motif-containing 21 antibodies; Agonistic antiAT1R - autoantibodies against the angiotensin II type 1 receptor; Agonistic anti-ETAR - autoantibodies against the endothelin-1 type A receptor; PAH - pulmonary arterial hypertension; ILD - interstitial lung disease

\section{CONCLUSION}

The outcomes of this review suggest that there are many molecules that can serve as biomarkers in the diagnosis, assessment of disease activity, and prediction of internal organs complications in SSc. Moreover, we may potentially classify patients into different risk groups, to improve treatment implementation and its efficacy. Nevertheless, advanced research including bigger groups of participants is needed for validation.

\section{REFERENCES}

1. Hasegawa M. Biomarkers in systemic sclerosis: Their potential to predict clinical courses. J Dermatol. 2016;43(1):29-38.

2. Knobler R, Moinzadeh $P$, Hunzelmann N et al. European Dermatology Forum S1-guideline on the diagnosis and treatment of sclerosing diseases of the skin, Part 1: localized scleroderma, systemic sclerosis and overlap syndromes. J Eur Acad Dermatol Venereol. 2017;31(9):1401-24.

3. Matsushita T, Takehara K. An update on biomarker discovery and use in systemic sclerosis. Expert Rev Mol Diagn. 2017;17(9):823-33.

4. Denton CP, Wells AU, Coghlan JG. Major lung complications of systemic sclerosis. Nat Rev Rheumatol. 2018;14(9):511-27.

5. Walecka I. Systemic sclerosis and the gastrointestinal tract. Prz Gastroenterol. 2017;12(3):163-8.

6. Kumar S, Singh J, Rattan S et al. Review article: pathogenesis and clinical manifestations of gastrointestinal involvement in systemic sclerosis. Aliment Pharmacol Ther. 2017;45(7):883-98.

7. Rosato E, Gigante A, Barbano B et al. Prognostic Factors of Renal Involvement in Systemic Sclerosis. Kidney Blood Press Res. 2018;43(3):682-9.
8. Bosello S, De Luca G, Berardi G et al. Cardiac troponin T and NT-proBNP as diagnostic and prognostic biomarkers of primary cardiac involvement and disease severity in systemic sclerosis: A prospective study. Eur J Intern Med. 2019;60:46-53.

9. Chaisson NF, Hassoun PM. Systemic Sclerosis-Associated Pulmonary Arterial Hypertension. CHEST. 2013;144(4):1346-56.

10. Matsuda KM, Yoshizaki A, Kotani Hetal. Serum heat shock protein 27 levels in patients with systemic sclerosis: a possible biomarker of skin sclerosis. J Eur Acad Dermatol Venereol. 2020. doi.org/10.1111/jdv. 16885

11. Liu Q, Lin J, Han J et al. Immunoglobulin $G$ galactosylation levels are decreased in systemic sclerosis patients and differ according to disease subclassification. Scand J Rheumatol. 2020;49(2):146-53.

12. Arakawa $\mathrm{H}$, Jinnin M, Muchemwa FC et al. Adiponectin expression is decreased in the involved skin and sera of diffuse cutaneous scleroderma patients. Experimental dermatology. 2011;20(9):764-6.

13. Zolkiewicz J, Stochmal A, Rudnicka L. The role of adipokines in systemic sclerosis: a missing link? Arch Dermatol Res. 2019;311(4):251-63.

14. Kawabata K, Makino T, Makino K et al. IL-16 expression is increased in the skin and sera of patients with systemic sclerosis. Rheumatology (0xford). 2020;59(3):519-23.

15. Farina G, Lafyatis D, Lemaire R et al. A four-gene biomarker predicts skin disease in patients with diffuse cutaneous systemic sclerosis. Arthritis Rheum. 2010;62(2):580-8.

16. Wermuth PJ, Piera-Velazquez S, Rosenbloom J et al. Existing and novel biomarkers for precision medicine in systemic sclerosis. Nat Rev Rheumatol. 2018;14(7):421-32.

17. Rice LM, Ziemek J, Stratton EA et al. A longitudinal biomarker for the extent of skin disease in patients with diffuse cutaneous systemic sclerosis. Arthritis Rheumatol. 2015;67(11):3004-15. 
18. Abd-Elmawla MA, Hassan M, Elsabagh YA et al. Deregulation of long noncoding RNAs ANCR, TINCR, HOTTIP and SPRY4-IT1 in plasma of systemic sclerosis patients: SPRY4-IT1 as a novel biomarker of scleroderma and its subtypes. Cytokine. 2020;133:155124.

19. Lammi MR, Saketkoo LA, Okpechi SC et al. Microparticles in systemic sclerosis: Potential pro-inflammatory mediators and pulmonary hypertension biomarkers. Respirology. 2019;24(7):675-83.

20. Elhai M, Hoffmann-Vold AM, Avouac J et al. Performance of Candidate Serum Biomarkers for Systemic Sclerosis-Associated Interstitial Lung Disease. Arthritis Rheumatol. 2019;71(6):972-82.

21. Wu M, Baron M, Pedroza C et al. CCL2 in the Circulation Predicts LongTerm Progression of Interstitial Lung Disease in Patients With Early Systemic Sclerosis: Data From Two Independent Cohorts. Arthritis Rheumatol. 2017;69(9):1871-8.

22. van der Kroef M, Carvalheiro T, Rossato M et al. CXCL4 triggers monocytes and macrophages to produce PDGF-BB, culminating in fibroblast activation: Implications for systemic sclerosis. J Autoimmun. 2020;111:102444.

23. Elhai M, Avouac J, Hoffmann-Vold AM et al. OX40L blockade protects against inflammation-driven fibrosis. Proc Natl Acad Sci U S A. 2016;113(27):E3901-10.

24. van den Hoogen F, Khanna D, Fransen J et al. 2013 classification criteria for systemic sclerosis: an American College of Rheumatology/European League against Rheumatism collaborative initiative. Arthritis Rheum. 2013;65(11):2737-47.

25. Mohan C, Assassi S. Biomarkers in rheumatic diseases: how can they facilitate diagnosis and assessment of disease activity? BMJ. 2015;351:h5079.

26. Wodkowski M, Hudson M, Proudman S et al. Monospecific anti-R052/ TRIM21 antibodies in a tri-nation cohort of 1574 systemic sclerosis subjects: evidence of an association with interstitial lung disease and worse survival. Clin Exp Rheumatol. 2015;33(4 Suppl 91):S131-5.

27. Becker M0, Kill A, Kutsche M et al. Vascular receptor autoantibodies in pulmonary arterial hypertension associated with systemic sclerosis. Am J Respir Crit Care Med. 2014;190(7):808-17.

28. JaegerVK, Wirz EG, Allanore Y et al. Incidences and Risk Factors of Organ Manifestations in the Early Course of Systemic Sclerosis: A Longitudinal EUSTAR Study. PLoS One. 2016;11(10):e0163894.

29. Marie I, Leroi AM, Menard JF et al. Fecal calprotectin in systemic sclerosis and review of the literature. Autoimmun Rev. 2015;14(6):547-54.

30. Caimmi C, Bertoldo E, Venturini A et al. Relationship Between Increased Fecal Calprotectin Levels and Interstitial Lung Disease in Systemic Sclerosis. J Rheumatol. 2019;46(3):274-8.

31. Stern EP, Guerra SG, Chinque H et al. Analysis of anti-RNA polymerase III antibody positive systemic sclerosis suggests altered GPATCH2L and CTNND2 expression in scleroderma renal crisis. J Rheumatol. 2020. doi: 10.3899/jrheum.190945. [Online ahead of print].

32. Liu C, Hou Y, Xu D et al. Analysis of anti-RNA polymerase III antibodies in Chinese Han systemic sclerosis patients. Clin Rheumatol. 2020;39(4):1191-7.

33. Okrój M, Johansson M, SaxneT et al. Analysis of complement biomarkers in systemic sclerosis indicates a distinct pattern in scleroderma renal crisis. Arthritis Res Ther. 2016;18(1):267.

34. Rice LM, Mantero JC, Stratton EA et al. Serum biomarker for diagnostic evaluation of pulmonary arterial hypertension in systemic sclerosis. Arthritis Res Ther. 2018;20(1):185.
35. Hickey PM, Lawrie A, Condliffe R. Circulating Protein Biomarkers in Systemic Sclerosis Related Pulmonary Arterial Hypertension: A Review of Published Data. Front Med (Lausanne). 2018;5:175.

36. Allanore Y, Komocsi A, Vettori S et al. N-terminal pro-brain natriuretic peptide is a strong predictor of mortality in systemic sclerosis. Int J Cardiol. 2016;223:385-9.

37. Izumiya $Y$, Jinnn M, Kimura Y et al. Expression of Let-7 family microRNAs in skin correlates negatively with severity of pulmonary hypertension in patients with systemic scleroderma. Int J Cardiol HeartVasc. 2015;8:98-102.

38. Sun Q, Hackler J, Hilger J et al. Selenium and Copper as Biomarkers for Pulmonary Arterial Hypertension in Systemic Sclerosis. Nutrients. 2020;12(6).

39. Senturk B, Akdeniz B, Yilmaz MB et al. Whole blood viscosity in systemic sclerosis: a potential biomarker of pulmonary hypertension? Clin Rheumatol. 2020;39(1):49-56.

40. Chrabaszcz M, Małyszko J, Sikora M et al. Renal Involvement in Systemic Sclerosis: An Update. Kidney Blood Press Res. 2020;45(4):532-548.

\section{ACKNOWLEDGMENTS}

This research was funded by the Polish Ministry of Science and Higher Education, grant number MNiSW/2020/220/DIR/NN4.

\section{ORCID and contributionship}

Katarzyna Karina Pawlik - 0000-0003-4342-4324 A, B, D-F

Anna Bohdziewicz - 0000-0001-7012-2298 D-F

Magdalena Chrabąszcz - 0000-0003-1009-6395 D-F

Anna Stochmal - 0000-0003-4447-282X E-F

Mariusz Sikora - 0000-0002-6162-9916 E-F

Rosanna Alda-Malicka - 0000-0002-3815-7842 E-F

Joanna Czuwara - 0000-0001-6139-7281 E-F

Lidia Rudnicka - 0000-0002-8308-1023 E-F

\section{Conflict of interest}

Authors declare no conflict of interest.

\section{CORRESPONDING AUTHOR Joanna Czuwara \\ Department of Dermatology, Medical University of Warsaw Koszykowa 82A, 02-008, Warszawa, Poland \\ tel.: 506166153 \\ e-mail:jczuwara@gmail.com}

Received: 24.08 .2020

Accepted: 01.10 .2020

\footnotetext{
A - Work concept and design, B - Data collection and analysis, C - Responsibility for statistical analysis, D -Writing the article, $\mathbf{E}$-Critical review, $\mathbf{F}$ - Final approval of the article
} 